\title{
Different Choices between Study and Working-A Case Study in the Application of Decision Support
}

\author{
Xiaobin $\mathrm{Li}^{1,2, *}$, Yaling $\mathrm{Zhu}^{1}$, Xiangwei $\mathrm{Li}^{1}$ and Marko Bohanec ${ }^{3,4}$ \\ ${ }^{1}$ College of Software Engineering, Lanzhou Institute of Technology, 730050 Lanzhou, China \\ ${ }^{2}$ Jozef Stefan International Postgraduate School, 1000 Ljubljana, Slovenia \\ ${ }^{3}$ Jozef Stefan Institute, Jamova 39, 1000 Ljubljana, Slovenia \\ ${ }^{4}$ University of Nova Gorica, Vipavska 13, 5000 Nova Gorica, Slovenia \\ ${ }^{*}$ Corresponding author
}

\begin{abstract}
Coordinating the conflict of time between study and working is a common problem for many people who are employed by a company. Especially for those who want to be released from regular work for study for a long time. They may have to enter into contracts with various agencies and if their circumstances change, they could potentially find themselves in breach of contract and may have to reconsider their options. In this paper, we consider a scenario where a doctoral student found himself in such a predicament. Two models were built to help the subject to make his decision, which are a quantitative model built by TreeAge Pro and a qualitative model built by DEXi.
\end{abstract}

Keywords-decision support; decision making; quantitative; qualitative; TreeAge Pro; DEXi

\section{INTRODUCTION}

Multi-criteria decision aid (MCDA), also termed multicriteria decision analysis and multi-criteria decision making (MCDM), is a discipline which aims at supporting decision makers who are faced with making numerous and conflicting evaluations [1]. Many different MCDA methods have been implemented [2], some of these software programs develop quantitative decision models, and meanwhile the others build qualitative decision models to evaluate options in order to help decision makers making decision.

TreeAge Pro [3] is the leader in visual modeling tools, which builds and analyzes decision trees in manner of quantitative to study any kind of decision problem. TreeAge Pro has been used in different industries; DEXi [4] is a standalone computer program for multi-attribute decision making. It facilitates interactive development of qualitative multiattribute and hierarchical decision models and the evaluation of options. DEXi has been used in many real-life decision problems in the areas such as selection and evaluation of computer hardware and software, evaluation of companies and business partners, personnel management, project evaluation, land-use planning, risk assessment in medicine and health-care [5].

In this work, we apply TreeAge Pro and DEXi to analyze a decision making problem in which a doctoral student facing with different choices between working and studying. The problem is described blow.
The student is in his first year of four years of doctoral studies in an institute far from the company where he works in. Therefore, he has to manage his study full time. He encounters some problems regarding contracts with different agencies and subsequently needs to make some decisions.

In October 2013, the subject received an enrollment letter from one university for a three years master's degree studying in the city where he is currently carrying out his doctoral studying. He had also signed a contract with his company which he was working for in his city of origin. The contract states the company will reserve his position for up to three years, until October 2016, when he receives his master's degree and he has to return to work for the company and receive a basic salary for the previous three years totaling $\$ 18,000$; averaging $\$ 6,000$ per year. If he fails his master's degree because of subjective reasons before October 2016, the company will not pay him any salary, and he must return to work for the company, otherwise it means he must give up his job and must repay the company $\$ 36,000$ as damage for breach of contract.

On commencement of his university studies in October 2013, the student found it difficult to study as he had a problem with the language. The university recommended him to study in another international school where all courses would be given in English. However, that school only offers four years of doctoral study programs. He contacted his company and the director agreed to allow him change the study program and sign another contract when he would have time to go back to the company during studying period; so far he has never had enough time to go back to his city to sign the contract. In the meantime, he won a scholarship of $\$ 6,000$ per year from an agency (Agency A) in the city where he was studying on the condition that he successfully completes his doctoral studies within a maximum of five years; otherwise, he must refund the scholarship.

In the first school year, he was studying English to obtain a qualification which the school required. His doctoral studying started in October 2014. Meanwhile he got a yearly scholarship from Agency A.

After two years in 2015, the director of the company changed and he made contact with the new director about his contract of study. He was advised to keep the original contract, which means the company states he must go back to work in 
October 2016 at the latest. At this time, he would have done his doctoral studying for two years with the support of the scholarship from agency A. However, he will still have an opportunity to renegotiate to extend the time of his study with his company. But the results are nonetheless unpredictable.

In 2014, he introduced his brother to work in a company in the city where he studies and if leaves the city, his brother cannot work there without him remaining resident in the city. Thus his leaving will lead to his brother's loss of salary in the sum of $\$ 1,000$. 2016.

Therefore, he will need to make a decision in October

In this paper, the second chapter describes three alternatives for the student; the third chapter performs the financial analysis of three alternatives by using of the quantitative tool TreeAge Pro; the fourth chapter carries out the qualitative analysis with $\mathrm{DEXi}$; while the final chapter contains the conclusion received from this work.

\section{ALTERNATIVES}

There are three choices for the student to select which are explained as follows.

\section{A. Modify Contract}

To contact the company before October 2016, and negotiate with the company to extend his contract of study, there will be three different cases described below.

\section{1) Extend contract till 2019}

If the contract can be extended till October 2019, the probability of this situation is 0.25 estimated by the subject, meanwhile the company will reserve his position, it will be beneficial for the student as he will have enough time of five years to successfully obtain a doctoral degree, there will be two possibilities in this case:

- Get PhD with the probability of 0.90 ; in this case, he will get $\$ 36,000$ of salary from his company for the past six years.

- Not get PhD with the probability of 0.10 , he has to repay $\$ 30,000$ of five years of scholarship to Agency A, meanwhile he cannot get $\$ 36,000$ of salary from his company for the past six years, which means $\$ 66,000$ of loss in total. The word "data" is plural, not singular.

\section{2) Extend contract till 2018}

If the contract can be extended till October 2018 with the probability of 0.50 , allowing him four years to finish his doctoral studying, there will be also two possibilities in this case similar to the above case which is shown in Figure I.

\section{3) Fail to extend contract}

If the contract with his company will not be extended with the probability of 0.25 , this will lead to two possible results:

- Breach of contract with the probability of 0.90 since the subject is eager to get a $\mathrm{PhD}$; in this case, he cannot get $\$ 36,000$ of salary from his company for the past six years if he could pass responsibility of the contract with his company, otherwise he has to pay the company $\$ 72,000$ as penalty of breach of contract.

- Quit studying with the probability of 0.10 . In this case, the subject will loss $\$ 49,000$ which is the sum of his brother's $\$ 1,000$ loss of salary, $\$ 12,000$ of repayment to Agency A of two years scholarship without finishing $\mathrm{PhD}$, and $\$ 36,000$ of loss of salary without any degree received in three years of studying.

\section{B. Breach of Contract}

If the company will not agree to modify the contract, and the student wants to continue studying, then he must breach of contract. This case leads to the same economic result to the subject as described above when the contract fails to be extended and he has to breach of contract.

\section{Quit Studying}

If the student wants to retain his job, and the company will not agree to extend the contract, then he has to quit studying. This case leads to the same economic result to the subject as described above when the contract fails to be extended and he has to quit studying.

\section{FINANCIAL ANALYSIS}

As the subject stated, the financial factor plays an important role when he makes a decision on this issue, therefore a financial analysis was done by using the tool TreeAge Pro.

\section{A. Structure}

Concerning the three options, the following structure was built by TreeAge Pro to analyze the problem for the student which is shown in figure I.

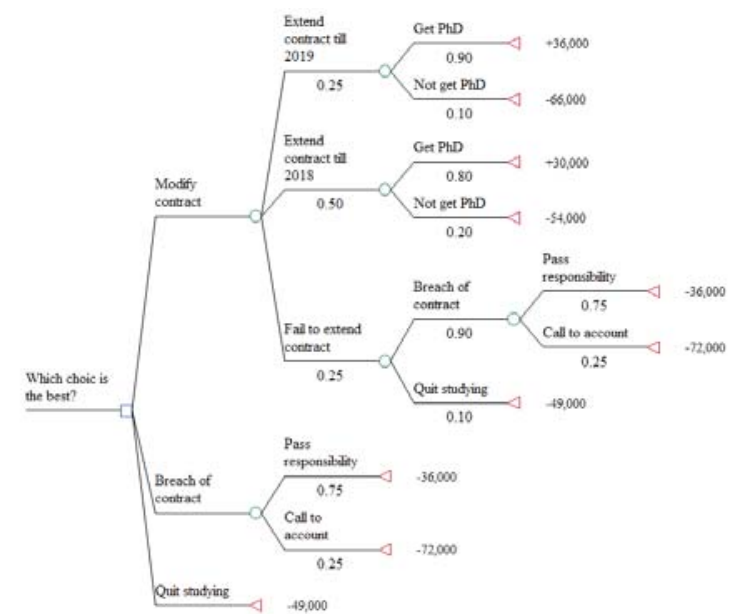

FIGURE I. THE STRUCTURE OF THE MODEL BUILT BY TREEAGE PRO

The number below line is the probability corresponds to the situation above, e.g. 0.50 means the probability of "Extend 
contract till 2018"; the positive number represents income the subject could get in corresponding situation, e.g. $+36,000$ means the subject could get $\$ 36,000$ in the situation of "Modify contract-Extend contract till 2019-Get PhD", which is the total amount of six years of salary from his company; the negative number represents the loss of the subject in corresponding situation, e.g. -66,000 means the subject could lose $\$ 66,000$ in the situation of "Modify contract-Extend contract till 2019- Not Get PhD", which is the sum of losses of six years of salary $\$ 36,000$ from his company and repayments of five years of scholarship $\$ 30,000$ to Agency A.

\section{B. Results}

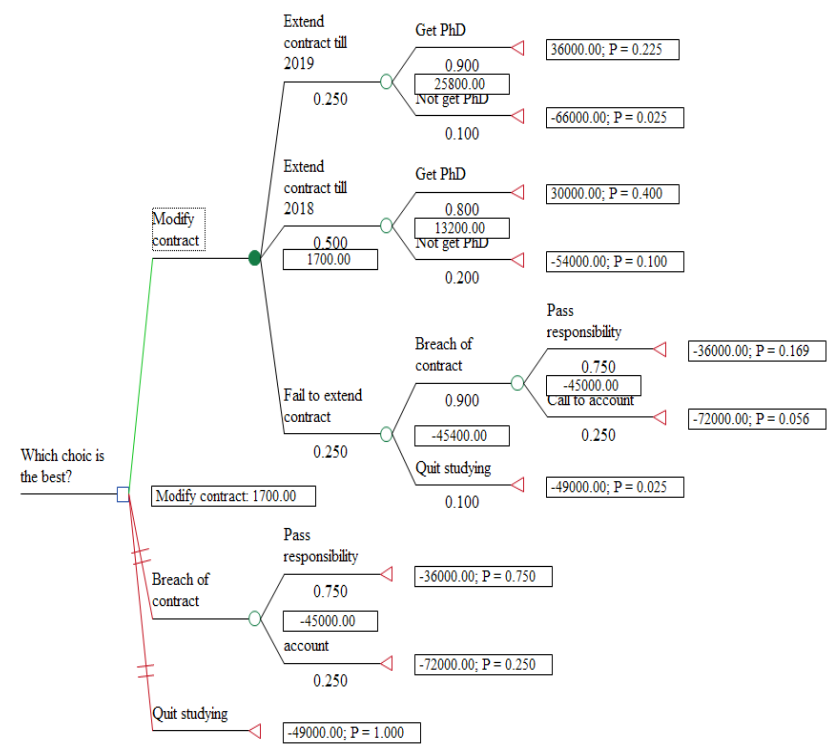

FIGURE II. THE BEST CHOICE FOR THE STUDENT FROM FINACIAL ASPECT

From the financial aspect, the best choice for the student is to "Modify contract" since he will get $\mathrm{PhD}$ with $\$ 1,700$ of income, while "Breach of contract" and "Quit studying" will lead to a loss of $\$ 45,000$ and $\$ 49,000$ respectively showing in figure II, which is created by using the instruction "Roll Back" under the menu "Analysis" from TreeAge Pro. The positive number in the boxes represent incomes the subject could get in corresponding situation, while the negative number represents the losses, and the value of $\mathrm{P}$ represents the probability of the corresponding situation.

\section{Analysis}

Figure III shows the rank of three choices, which is created by using the instruction "Rankings" under the menu "Analysis" from TreeAge Pro.

The chart shows, modifying contract is the best choice, breach of contract is the worse choice, while quitting studying is the worst choice for the subject from the perspective of economy.

Rankings
\begin{tabular}{|l|l|l|}
\hline Rank & Strategy & Value \\
\hline 1 & Modify contract & 1700.00 \\
\hline 2 & Breach of contract & -45000.00 \\
\hline 3 & Quit studying & -49000.00 \\
\hline
\end{tabular}
FIGURE III. RANK OF THREE CHOICES

\section{QUALITATIVE ANALYSIS}

The decision is not only based on the financial aspect but on some comprehensive factors as the student stated, below is the qualitative analysis done by using the tool DEXi.

\section{A. Attributes}

The qualitative analysis was performed from two viewpoints: the financial and satisfaction factors.

The Financial factor was concerned with two aspects of incomes and losses, both of them are divided into direct influence and potential influence. As for the incomes, the scales are high, medium and low decreasingly, while losses are low, medium, and high decreasingly.

The Satisfaction factor was concerned with three aspects involving career, living and family. Career means how the decision will influence the student's career in the future; living means how the decision will influence the student's living standards in the future; while family means how the decision will influence the family happiness.

The following model was built using DEXi which is shown in figure IV.

\begin{tabular}{ll} 
Attribute & Description \\
\hline CHOICE & Best choice for the student \\
-FINANCIAL & Influence to the student from financial aspect \\
-INCOMES & Positive influence to the student from financial aspect \\
-DIRECT INCOMES & Direct incomes \\
POTENTIAL INCOMES & Potential incomes in the future \\
LOSSES & Negative influence to the student from financial aspect \\
-DIRECT LOSSES & Direct losses \\
POTENTIAL LOSSES & Potential losses in the future \\
SATISFACTION & Satisfaction \\
-CAREER & Career in the future \\
-LIVING & Living standards in the future \\
FAMILY & Happiness of family
\end{tabular}

FIGURE IV. THE STRUCTURE OF THE MODEL BUILT BY DEXI

\section{B. Decision Rules}

\begin{tabular}{lll}
\multicolumn{2}{l}{ FINANCIAL } & SA TISFACTION \\
\hline $56 \%$ & $44 \%$ & \\
\hline 1 unacc & $*$ & unacc \\
$2{ }^{*}$ & unacc & unacc \\
\hline 3 acc & $>=$ acc & acc \\
\hline $4>=$ good & acc & good \\
\hline $5>=$ good & $>=$ good & exe \\
\hline
\end{tabular}

FIGURE V. PART OF THE DECISION RULES

As the subject stated, if any of the factors are unacceptable, then the result is unacceptable (rule 1 and 2); financial factor is slightly more important $(56 \%)$ than the other factors, the potential financial influence is more important than direct influence. As for the satisfaction factor, career is slightly more 
important than family happiness and living standards, while family happiness is slightly more important than living standards. According to the subject's criteria, the decision rules are defined and part of the decision rules are shown in figure $\mathrm{V}$.

\section{Results}

The evaluation results are shown in figure VI.

Evaluation results
\begin{tabular}{llll} 
& & & \\
Attribute & Modify contract & Breach of contract & Quit studying \\
\hline CHOICE & good & acc & unacc \\
FINANCIAL & exe & acc & good \\
-INCOMES & high & medium & low \\
-DIRECT INCOMES & high & low & low \\
POTENTIAL INCOMES & high & high & low \\
LOSSES & low & medium & low \\
-DIRECT LOSSES & low & high & high \\
POTENTIAL LOSSES & low & low & medium \\
SATISFACTION & acc & acc & unacc \\
-CAREER & exe & exe & unacc \\
-LIVING & acc & good & acc \\
FAMILY & acc & acc & good
\end{tabular}

FIGURE VI. THE EVALUATION RESULTS BY DEXI

Figure VI shows, to modify the contract is the best choice for the student; to breach of contract is also an acceptable choice; but to quit studying is not acceptable.

\section{Analysis}

\section{1) Selective explanation}

Figure VII shows the results of selective explanation.

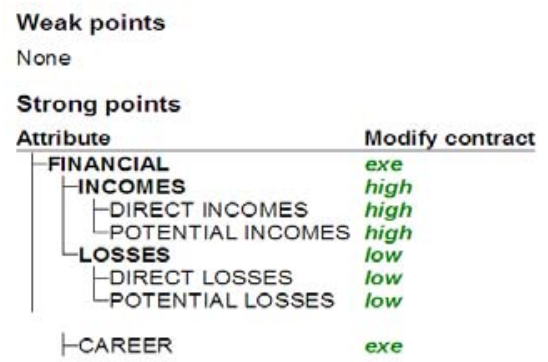

FIGURE VII. THE RESULTS OF SELECTIVE EXPLANATION BY DEXI

Figure VII shows that to modify the contract is the best choice. According to the subject's criteria, to extend the contract will lead to excellent financial results and he will have excellent career in the future.

\section{2) +-1 Analysis}

Figure VIII shows the results of plus-minus-1 analysis. This shows that, when the family happiness decreases from acceptable to unacceptable, then the decision result will be unacceptable; when the direct incomes increase from low to medium, the potential losses decrease from high to medium, therefore the result will become "good".

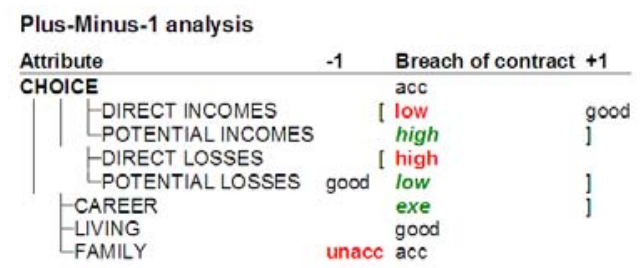

FIGURE VIII. THE RESULTS OF PLUS-MINUS-1 ANALYSIS BY DEXI

\section{CONCLUSION}

In this study a quantitative model and a qualitative model have been built which were implemented in the TreeAge Pro and DEXi environment respectively. Three different choices have been analyzed systematically by means of techniques supplied by TreeAge Pro and DEXi. The choices are: modifying the contract, breach of contract, or quitting study. It was found that to modify the contract is the best choice for the student; breach of contract is also an acceptable choice, but to quit studying is not acceptable.

From the results it could be seen that all choices may possibly lead to financial loss because a doctoral degree requires investment of both money and time. If the student achieves a PhD successfully, the potential benefit to the student is considerable from a variety of aspects, not only because of the financial advantages but also due to enhanced career prospects, living standards, and family happiness. Hence the purpose of this study is to help the subject to find the best option with minimum losses.

The original aim of the project was to identify the various factors influencing the decision that the student had to make and after analyzing them, assist him in making an informed decision. As there are a number of factors outside the control of the student the final decision will depend on the circumstances that prevail at the time that the decision has to be made.

\section{ACKNOWLEDGMENT}

The paper is supported by the Gansu construction scientific research projects of china under grant No.0612-05 and the LanZhou special project of strategic new industry technology No.2013-4-23.

\section{REFERENCES}

[1] V. Belton and T. J. Stewart, Multiple Criteria Decision Analysis: An Integrated Approach. Boston: Kluwer Academic Publishers, 2002.

[2] D. T. Maxwell, "Decision analysis: find a tool that fits," OR/MS Today, October 2008.

[3] TreeAge, 2015.

[4] M. Bohanec, DEXi: Program for Multi-Attribute Decision Making, User's Manual, Version 3.00, 2008.

[5] I. Lipušček, M. Bohanec, L. Oblak, L. Zadnik Stirn, "A multi-criteria decision-making model for classifying wood products with respect to their impact on environment," International Journal on Life Cycle Assessment, vol. 15(4), pp. 359-367, 2010. 